\title{
Mycoplasma orale
}

National Cancer Institute

\section{Source}

National Cancer Institute. Mycoplasma orale. NCI Thesaurus. Code C86598.

A species of anaerobic, Gram-negative, pseudo-cocci shaped bacteria assigned to the phylum Firmicutes. This species is nonmotile, urease negative, does not hydrolyze carbohydrates, gelatin, or casein, and is sensitive to penicillin, erythromycin and aminoglycosides. M. orale is commensal in the human oropharynx and is usually nonpathogenic, but can cause infections in immunosuppressed patients and is a common contaminant found in cultured cells. 